\title{
Differentiation of Alzheimer Conditions in MR Brain Images Using a Single Inception Module Network
}

\author{
Sreelakshmi SHAJI ${ }^{\mathrm{a}, 1}$, Nagarajan GANAPATHY ${ }^{\mathrm{b}}$ and Ramakrishnan \\ SWAMINATHAN ${ }^{\mathrm{a}}$ \\ a Biomedical Engineering Group, Department of Applied Mechanics, Indian Institute of \\ Technology Madras, Chennai, India \\ ${ }^{\mathrm{b}}$ Peter L. Reichertz Institute for Medical Informatics of TU Braunschweig and \\ Hannover Medical School, Germany
}

\begin{abstract}
In this study, an attempt has been made to differentiate Alzheimer's Disease (AD) stages in structural Magnetic Resonance (MR) images using single inception module network. For this, T1-weighted MR brain images of $\mathrm{AD}$, mild cognitive impairment and Normal Controls (NC) are obtained from a public database. From the images, significant features are extracted and classified using an inception module network. The performance of the model is computed and analyzed for different input image sizes. Results show that the single inception module is able to classify $\mathrm{AD}$ stages using MR images. The end-to-end network differentiates $\mathrm{AD}$ from $\mathrm{NC}$ with $85 \%$ precision. The model is found to be effective for varied sizes of input images. Since the proposed approach is able to categorize $\mathrm{AD}$ stages, single inception module networks could be used for the automated $\mathrm{AD}$ diagnosis with minimum medical expertise.
\end{abstract}

Keywords. Alzheimer's Disease, Mild cognitive impairment, Magnetic Resonance Imaging, Inception module

\section{Introduction}

Alzheimer's Disease (AD) is the most dominant type of dementia, leading to progressive and irreversible loss in cognitive abilities. It is estimated that by 2050,152 million populations worldwide will suffer from AD [1]. Due to the increasing prevalence, accurate diagnosis of the prodromal stage of $\mathrm{AD}$, Mild Cognitive Impairment (MCI), is crucial for delaying the progression of the disease [2].

The brain atrophy associated with AD is considered to be a significant biomarker in characterizing the disease progression [3]. Structural Magnetic Resonance Imaging (sMRI) is a widely preferred non-invasive neuroimaging technique for analyzing the tissue losses in the brain regions. Based on sMRI estimates, several Computer-Aided Decision support (CAD) systems have been developed for the early prediction of AD [2][3]. Some of the CAD approaches include k-nearest neighbor [3] and support vector

${ }^{1}$ Corresponding Author: Sreelakshmi Shaji, Biomedical Engineering Group, Department of Applied Mechanics, Indian Institute of Technology, Madras, Chennai, India, E-mail: lakshminair.here@gmail.com 
machine [2]. These methods employ handcrafted features which may not be suitable with subsequent classifiers, thus leading to reduction in the performance of the system.

Recently, Convolutional Neural Networks (CNNs) achieve competitive performance in classifying medical images. Due to the ability to perform task-specific feature extraction for constructing classifiers, CNNs have been widely utilized in developing $\mathrm{CAD}$ systems for $\mathrm{AD}$ diagnosis [10]. A deep CNN model has been used to differentiate AD stages from axial view MR brain images [8]. Hippocampal regions have been extracted from sMRI to train 2D CNN for AD prediction [4].

In this work, an analysis on the effect of a single inception module network in the automated differentiation of $\mathrm{AD}, \mathrm{MCI}$ and NC subjects using sMRI brain images has been carried out [5]. A single layer end-to-end inception network is developed for characterizing the AD stages with a limited training dataset. The performance of the model is computed and analyzed by tuning the size of input images.

\section{Methods}

\subsection{Image Database}

The T1-weighted brain MR images of $29 \mathrm{AD}, 63 \mathrm{MCI}$ and $92 \mathrm{NC}$ subjects are acquired from the Open Access Series of Imaging Studies (OASIS) cross-sectional database [6]. The subjects are divided into $\mathrm{AD}, \mathrm{MCI}$ and $\mathrm{NC}$ based on Clinical Dementia Rating. Out of 176 slices, $90^{\text {th }}$ slice in the sagittal and trans-axial view are considered in the study [2][3]. In this study, all the images are converted into grayscale format and are resized to $256 \times 256,128 \times 128$ and $64 \times 64$ to study the effect of size of the input image on the performance of the inception network.

\subsection{Network Architecture}

To extract features and differentiate AD, MCI and NC MR brain images, an inception network is employed, the pipeline of which is shown in Figure 1. The network comprises Convolutional (Conv) layers of different sizes, Maximum (Max) and average pooling layers, a Fully Connected Layer (FCL) and a softmax layer. In order to align the patches, inception architecture employs convolution filters of sizes $1 \times 1$ (Conv 1), 3 × 3 (Conv 3) and $5 \times 5$ (Conv 5) [5]. Rectified linear unit is used as the non-linear activation function in the proposed network. An FCL that connects all the neurons from the preceding layer is employed [5]. The outputs of the non-linear softmax activation are used as probability scores for classification.

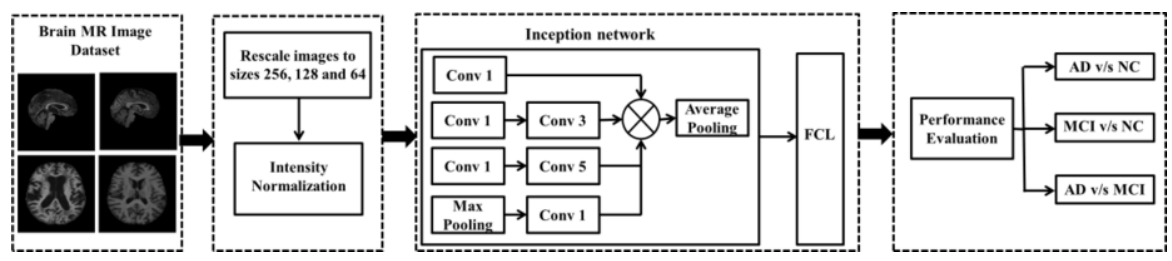

Figure 1. Block diagram of study

In this study, the inception network consists of three Conv layers and a Max pooling layer. Each Conv layer has varying number of filters such as 4, 8, 16, 24 and 32 and is all zero-padded. The Max pooling layer has a pool size and stride of 2 and 1 
respectively. These layers are concatenated which are further fed into an average pooling layer of size 3 . The number of epochs and batch size are fixed empirically to 50 and 10 respectively. An Adam optimizer with a learning rate of 0.01 is used.

The input images are split into training and testing set at a ratio of $80-20$. The network is trained and tested for three binary classification problems (AD v/s NC; AD $\mathrm{v} / \mathrm{s} \mathrm{MCI}$ and MCI v/s NC). The performance of the model is evaluated using performance matrices such as accuracy, recall, precision and F-measure [2][3].

\section{Results and Discussion}

The representative brain MR images of $\mathrm{AD}(\mathrm{a}, \mathrm{d}), \mathrm{MCI}(\mathrm{b}, \mathrm{e})$ and $\mathrm{NC}(\mathrm{c}, \mathrm{f})$ subjects are shown in Figure 2. It is observed that the white matter structures such as corpus callosum, brainstem and cerebellum are clearly visible in the sagittal view images. An enlargement in the lateral ventricles is observed in the trans-axial view images from $\mathrm{NC}$ to $\mathrm{AD}$ subjects. It is seen that the brain structures have different sizes and shapes as the diseases progresses.

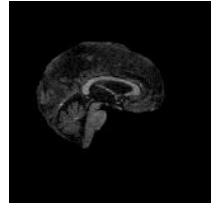

(a)

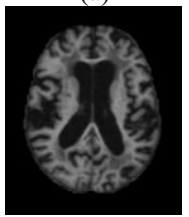

(d)

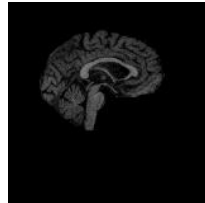

(b)

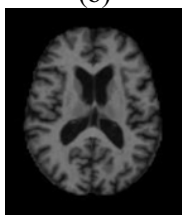

(e)

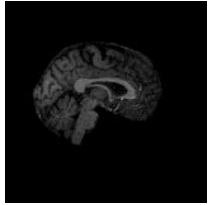

(c)

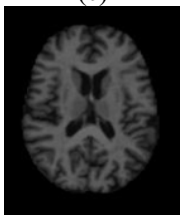

(f)

Figure 2. Representative MR brain images in sagittal (a-c) and trans-axial (d-f) view of AD (a,d), MCI (b,e) and $\mathrm{NC}(\mathrm{c}, \mathrm{f})$ subjects

The training accuracy and loss of the inception network for varying number of epochs using different input image sizes is shown in Figure 3 (a-c) and Figure 3 (d-f) respectively. It is seen from Figure 3 that for all the binary classifications, AD v/s NC $(\mathrm{a}, \mathrm{d}), \mathrm{MCI} \mathrm{v} / \mathrm{s} \mathrm{NC}(\mathrm{b}, \mathrm{e})$ and $\mathrm{AD} \mathrm{v} / \mathrm{s} \mathrm{MCI}(\mathrm{c}, \mathrm{f})$, the variations in the accuracy and training loss with number of epochs are maximum when image size is 256 and minimum for an image size of 64. It is found that the accuracy decreases with the decrease in image size and remains almost constant throughout the training process.

The diagnostic performance of the inception network in differentiating $\mathrm{AD} v / \mathrm{s} \mathrm{NC}$, $\mathrm{MCI}$ //s NC and AD v/s MCI for different input image sizes is tabulated in Table 1. It is seen that the accuracy and recall of the model in differentiating $\mathrm{AD}$ and $\mathrm{NC}$ remains similar when image sizes are 256 and 128 . For AD v/s MCI, the maximum accuracy is observed for an image size of 256. The approach exhibits maximum performance in differentiating $\mathrm{AD}$ and NC. A maximum performance of $70 \%$ is observed in differentiating MCI and NC when the image size is 128 . The least performance of model is obtained in categorizing AD and MCI. This could be due to subtle changes in the brain structures with disease progression. 


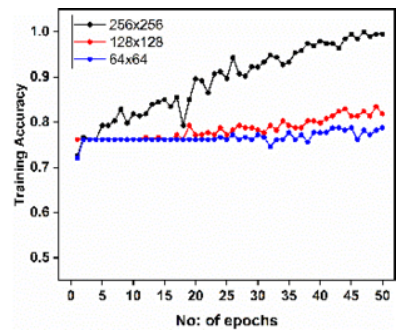

(a)

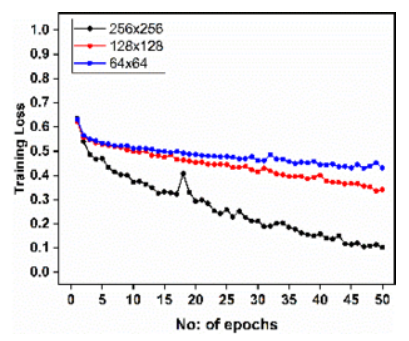

(d)

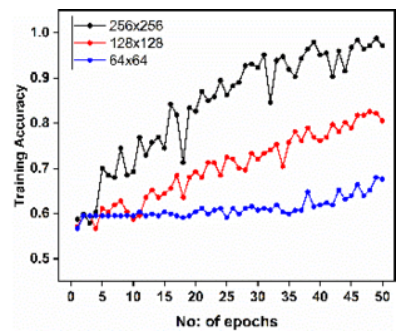

(b)

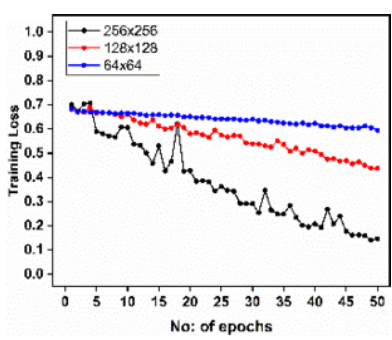

(e)

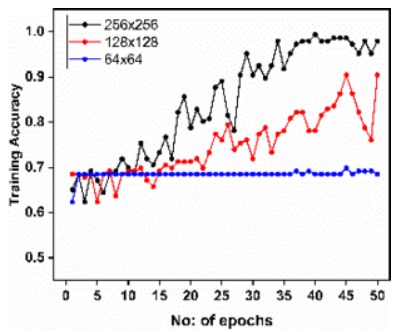

(c)

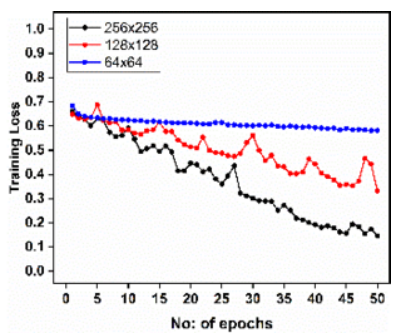

(f)

Figure 3. Variations in training accuracy and loss for varied number of epochs using different input image sizes $\mathrm{AD}$ v/s NC (a, d), MCI v/s NC (b, e) and AD v/s MCI (c, f)

Table 1. Performance (\%) of the proposed method in classifying AD v/s NC, MCI v/s NC and AD v/s MCI

\begin{tabular}{cccccc}
\hline Image size & Class & Accuracy & Precision & Recall & F-measure \\
\hline \multirow{3}{*}{$256 \times 256$} & AD v/s NC & 78 & 78 & 78 & $\mathbf{7 8}$ \\
& MCI v/s NC & 59 & 57 & 59 & 57 \\
& AD v/s MCI & $\mathbf{6 8}$ & $\mathbf{6 7}$ & $\mathbf{6 8}$ & $\mathbf{6 8}$ \\
\hline \multirow{5}{*}{$128 \times 128$} & AD v/s NC & 78 & 75 & 78 & 72 \\
& MCI v/s NC & $\mathbf{7 0}$ & $\mathbf{7 0}$ & $\mathbf{7 0}$ & $\mathbf{7 0}$ \\
\hline \multirow{5}{*}{$64 \times 64$} & AD v/s MCI & 58 & 54 & 58 & 55 \\
\hline & AD v/s NC & $\mathbf{8 2}$ & $\mathbf{8 5}$ & $\mathbf{8 2}$ & 77 \\
& MCI v/s NC & 60 & 59 & 60 & 53 \\
\hline
\end{tabular}

Table 2. Performance (\%) comparison of the proposed approach with the state-of-the-art methods

\begin{tabular}{llcccc}
\hline \multicolumn{1}{c}{ Author } & Class & Model & Learning & Accuracy & F-measure \\
\hline Islam and Zhang [7] & Multiclass & Deep CNN & FS & 73.75 & - \\
\hline Hon and Khan [8] & AD v/s NC & VGG16 & FS & 74.12 & - \\
\hline Puente-Castro et al. [9] & AD v/s NC & ResNet & TL & 86.47 & 32.07 \\
\hline \multirow{2}{*}{ Proposed method } & AD v/s NC & \multirow{2}{*}{ Single Layer Inception } & \multirow{2}{*}{ FS } & 82 & 77 \\
& MCI v/s NC & Network & & 68 & 70 \\
& AD v/s MCI & & & & 68 \\
\hline
\end{tabular}

FS - From Scratch; TL - Transfer Learning

F-measure is a widely used evaluation metric to assess the performance of binary classification problems. It is the harmonic mean of recall and precision and is a reasonable measure for the evaluation of imbalanced classes [10]. In this study, the proposed model achieves an F-measure of $78 \%, 70 \%$ and $68 \%$ in differentiating AD 
v/s NC, MCI v/s NC and AD v/s MCI respectively, validating the reliability of the model.

The performance of the proposed approach has been compared with the state-ofthe-art methods using OASIS database (see Table 2). Hon and Khan [8] have proposed a VGG16 trained model to classify AD from NC and obtained an accuracy of $74.12 \%$. Recently, transfer learning has been used to differentiate AD and NC [9].

A limitation of this study is that the parameters used for fine-tuning the network is based on random search method [11]. In future, optimization can be used to determine the tuning parameters. Also, the confidence of the model can be improved by using large number of clinical images with adaptive learning and cross-database training.

\section{Conclusion}

CNNs are widely used in $\mathrm{AD}$ prediction due to their ability to perform task-oriented feature extraction and classification. In this study, an end-to-end single layer inception module network has been developed for predicting AD stages. The key contribution of the study is to analyze the discriminative ability of the inception network in classifying $\mathrm{AD}$ stages using different sizes of MR images. The results indicate that the performance of the model varies with the image size. The model achieves highest performance in differentiating AD from NC. Thus single inception module networks could be used for the automated $\mathrm{AD}$ diagnosis with minimum medical expertise.

\section{References}

[1] ADI - Dementia statistics, Available at: https://www.alzint.org/about/dementia-facts-figures/dementiastatistics

[2] Anandh KR, Sujatha CM, Ramakrishnan S. A Method to Differentiate Mild Cognitive Impairment and Alzheimer in MR Images using Eigen Value Descriptors. J Med Syst. 2015 Nov 7;40(1):25.

[3] Ramaniharan AK, Manoharan SC, Swaminathan R. Laplace Beltrami eigen value based classification of normal and Alzheimer MR images using parametric and non-parametric classifiers. Expert Syst Appl. 2016 Oct 15;59:208-16.

[4] Aderghal K, Afdel K, Benois-Pineau J, Catheline G. Improving Alzheimer's stage categorization with Convolutional Neural Network using Transfer Learning and Different Magnetic Resonance Imaging Modalities. Heliyon. 2020 Dec 1;6(12):e05652.

[5] Szegedy C, Liu W, Jia Y, Sermanet P, Reed S, Anguelov D, et al. Going Deeper with Convolutions. 2014 Sep 16. Available at: http://arxiv.org/abs/1409.4842

[6] Marcus DS, Fotenos AF, Csernansky JG, Morris JC, Buckner RL. Open Access Series of Imaging Studies (OASIS): Longitudinal MRI Data in Nondemented and Demented Older Adults. J Cogn Neurosci. 2010 Dec;22(12):2677-84.

[7] Islam J, Zhang Y. A novel deep learning based multi-class classification method for Alzheimer's disease detection using brain MRI data. Proceedings of the International conference on brain informatics; 2017 Nov 16; Springer, Cham; p. 213-222.

[8] Hon M, Khan NM. Towards Alzheimer's disease classification through transfer learning. Proceedings of the International conference on bioinformatics and biomedicine; 2017 Nov 13; IEEE; p. 1166-1169.

[9] Puente-Castro A, Fernandez-Blanco E, Pazos A, Munteanu CR. Automatic assessment of Alzheimer's disease diagnosis based on deep learning techniques. Comput. Biol. Med. 2020 May 1;120:10376.

[10] Liu M, Xu C, Luo Y, Xu C, Wen Y, Tao D. Cost-sensitive feature selection by optimizing F-measures. IEEE Trans Image Process. 2017 Dec;27(3):1323-35.

[11] Victoria AH, Maragatham G. Automatic tuning of hyperparameters using Bayesian optimization. Evol Syst. 2020 May 25:1-7. 